\title{
Validation of self-reported and hospital-diagnosed atrial fibrillation: the HUNT study
}

Vegard Malmo ${ }^{1,2}$

Arnulf Langhammer ${ }^{3}$

Kaare H Bønaa ${ }^{2,3,4}$

Jan P Loennechen ${ }^{1,2}$

Hanne Ellekjaer ${ }^{5,6}$

'Department of Circulation and Medical Imaging, Norwegian University of Science and Technology, ${ }^{2}$ Department of Cardiology, St Olav's Hospital, ${ }^{3}$ Department of Public Health and General Practice, Norwegian University of Science and Technology, Trondheim, ${ }^{4}$ Department of Community Medicine, UiT

The Arctic University of Norway, Tromso, ${ }^{5}$ Stroke Unit, Department of Internal Medicine, St Olav's Hospital, ${ }^{6}$ Department of Neuroscience, Norwegian University of Science and Technology, Trondheim, Norway
Correspondence: Vegard Malmo Department of Circulation and Medical Imaging, Norwegian University of Science and Technology, PO Box 8905, 749I

Trondheim, Norway

Tel +4772829756

Fax +4773867966

Email vegard.malmo@ntnu.no
This article was published in the following Dove Press journal:

Clinical Epidemiology

II June 2016

Number of times this article has been viewed

Background: Self-reported atrial fibrillation (AF) and diagnoses from hospital registers are often used to identify persons with AF. The objective of this study was to validate self-reported AF and hospital discharge diagnoses of AF among participants in a population-based study.

Materials and methods: Among 50,805 persons who participated in the third survey of the HUNT Study (HUNT3), 16,247 participants from three municipalities were included. Individuals who reported cardiovascular disease, renal disease, or hypertension in the main questionnaire received a cardiovascular-specific questionnaire. An affirmative answer to a question on physician-diagnosed AF in this second questionnaire defined self-reported AF diagnoses in the study. In addition, AF diagnoses were retrieved from hospital and primary care (PC) registers. All AF diagnoses were verified by review of hospital and PC medical records.

Results: A total of 502 HUNT3 participants had a diagnosis of AF verified in hospital or PC records. Of these, 249 reported their AF diagnosis in the HUNT3 questionnaires and 370 had an AF diagnosis in hospital discharge registers before participation in HUNT3. The sensitivity of self-reported AF in HUNT3 was 49.6\%, specificity 99.2\%, positive predictive value (PPV) $66.2 \%$, and negative predictive value (NPV) $98.4 \%$. The sensitivity of a hospital discharge diagnosis of AF was $73.7 \%$, specificity $99.7 \%$, PPV 88.5\%, and NPV 99.2\%.

Conclusion: Use of questionnaires alone to identify cases of AF has low sensitivity. Extraction of diagnoses from health care registers enhances the sensitivity substantially and should be applied when estimates of incidence and prevalence of AF are studied.

Keywords: atrial fibrillation, epidemiology, diagnosis, questionnaire, validation

\section{Introduction}

Atrial fibrillation (AF) affects $2 \%-3 \%$ of the adult population, and has a major impact on mortality, morbidity, and quality of life for patients, and cost for the health care system. ${ }^{1-3}$ The prevalence has been increasing over the last few decades, primarily due to an aging population. The age-adjusted AF incidence is also rising, probably due to an increased prevalence of obesity and other cardiovascular risk factors in the population. ${ }^{4-7}$

Self-reported AF and diagnoses from hospital registers are the two most common sources used in epidemiological studies to identify persons with AF. Validation of these approaches has mainly been performed by confirming the AF diagnoses by review of electrocardiograms (ECGs), and written documentation in hospital records. ${ }^{8}$ Few studies have searched for AF cases missed by these methods and a very few have studied primary care (PC) records. To our knowledge, no studies have compared self-reported and hospital-diagnosed AF. 
The primary aim of this study was to validate self-reported and hospital-diagnosed AF among participants in a large population-based study, and compare those methods using diagnoses verified by a systematic review of medical records in hospitals and PC centers as a standard. The secondary aim was to evaluate the effects of sex, age, and type of AF on the results.

\section{Materials and methods}

During 2006-2008, all persons living in the county of NordTrøndelag aged 20 years or older $(n=93,860)$ were invited to the third survey of the HUNT Study (HUNT3). Fifty-four percent $(\mathrm{n}=50,805)$ of those eligible attended. ${ }^{9}$ We included participants living in three municipalities (Steinkjer, Verdal, and Inderøy) which have a representative mixture of urban and rural areas, and the same medical record software in general practice. Fifty-six percent $(n=16,247)$ of the eligible population in the three municipalities attended. The study was approved by the regional medical ethics research committee, REC Central (Id: 2012/151/REK midt) and written informed consent was obtained from all participants.

\section{Questionnaires}

A general questionnaire (Q1) was included in the letter of invitation. Attending participants who reported having had myocardial infarction, angina pectoris, stroke, renal disease, heart failure, hypertension, or other heart diseases were given a second questionnaire about cardiovascular diseases (cardiovascular-specific questionnaire [QCVD]) which included the following question: "Has a doctor told you that you have atrial fibrillation?" Of the 16,247 participants from the three municipalities, 4,142 received the QCVD and 3,536 (85.4\%) returned it in a prepaid envelope. Among the 3536 who returned the QCVD, 2,996 (84.7\%) answered the AF question, 376 responded yes, 2,620 responded no (Figure 1).

\section{Retrieval of AF diagnoses from registers in hospitals and PC centers}

To identify cases of AF among the 16,247 persons in the three study municipalities, their Norwegian personal identification numbers were linked to AF diagnoses in registers in hospitals and PC centers.

There are two hospitals in the county, in the cities of Levanger and Namsos. Norwegian hospital discharge registers include dates of admission and discharge for inpatient treatment and day of visit for outpatient treatments. Diagnoses are coded as primary or contributory for all visits and have been registered electronically since 1988 , initially using International Classification of Diseases version 9 (ICD-9) and version 10 (ICD-10) from 1999 until present. ICD-9 code 427.3

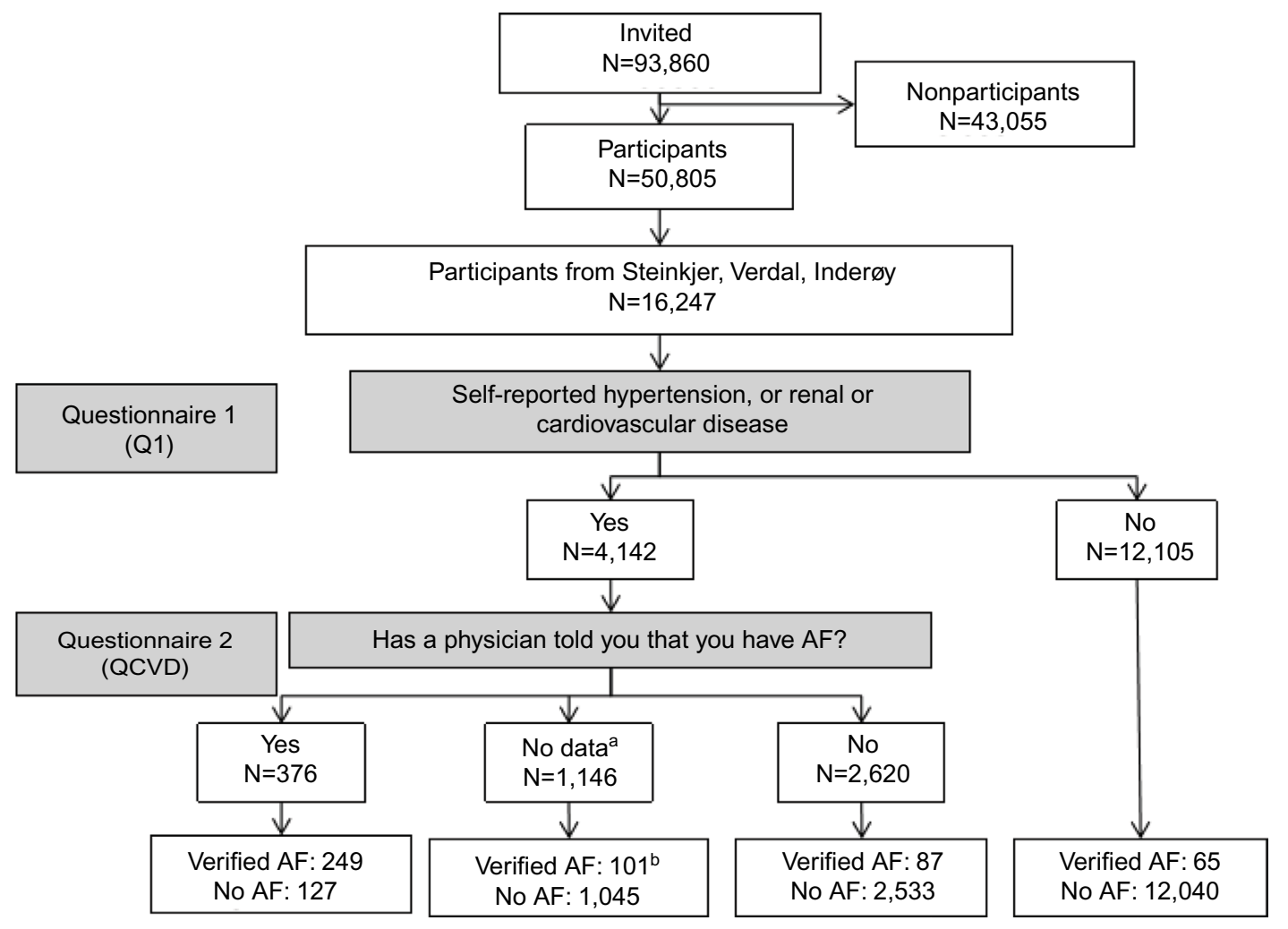

Figure I Flowchart on how the verified diagnoses of AF were found according to answers in the questionnaires.

Notes: ${ }^{a} 606$ participants did not return the second questionnaire and 540 of those who returned it did not answer the AF question. ${ }^{b} 59$ had received the second questionnaire but not returned it, 42 had returned it but not answered the AF question.

Abbreviations: AF, atrial fibrillation; QCVD, cardiovascular-specific questionnaire. 
and ICD-10 code I48 were used to identify AF patients. We extracted data from 1988 until 12 months after participation in HUNT3 from both hospitals in the area. For all participants with an AF diagnosis, we extracted clinical information and ECG data from the electronic medical records.

In the three study municipalities, there are 35 general practitioners at nine different medical centers. Registers in PC centers are administered locally at each center. All visits are coded with at least one International Classification of Primary Care (ICPC) code. ICPC code K78 “Atrial fibrillation/atrial flutter" was used to identify persons with AF. In addition to the diagnosis register, each patient has an electronic medical record that includes clinical information from each visit at the general practitioner. There were no other relevant health care providers in the county of Nord-Trøndelag at the study time. Relevant health care providers outside the county are St Olav's Hospital and private cardiologists in Trondheim. St Olav's Hospital is situated in the neighboring county, and treats more complex patients referred from the local hospitals. A medical report for all inpatient and outpatient admissions at St Olav's Hospital and visits to the private cardiologists is sent to the local hospital and the patient's general practitioner and thus available for the medical record review conducted.

Persons with either self-reported AF in HUNT3 or a diagnosis of AF or atrial flutter in hospital or PC registers were included for further validation by review of medical records from hospitals and PC centers.

"Verified AF" was defined as an ECG that could be classified as AF or atrial flutter according to standard criteria. ${ }^{3}$ If an ECG was unavailable, the medical record was reviewed for a written interpretation. When a physician had described the ECG as AF or atrial flutter using standard criteria, the case was defined as verified AF. Only participants with physician-diagnosed AF before the date of participation in HUNT3 were included in this study.

For 17 participants, the information in medical records was insufficient for classification. In these cases, two physicians, one specialist in cardiology (JPL) and one specialist in internal medicine (HE), evaluated the available information separately, grouping them into two categories: "probable AF" and "not likely AF". In case of disagreement, the patient was put in the most likely category after a consensus meeting. The eleven "probable AF" cases were included as verified diagnoses in the study.

Patients were also classified as having permanent or nonpermanent, ie, paroxysmal or persistent AF. ${ }^{10}$ For 19 persons, the documentation in medical records was insufficient to perform a reasonable classification.
Participants without self-reported AF were considered as not having AF if there was no diagnosis of AF either in hospitals or in PC centers. To detect possible AF cases not included in the registers (false-negative AF), we reviewed the hospital and $\mathrm{PC}$ records of 500 randomly selected study participants without self-reported AF or an AF diagnosis in the registers.

\section{Statistics}

All participants with a verified diagnosis of AF before participation in HUNT3 were included in validation analyses of the questionnaires and the hospital discharge diagnoses of AF. Participants with a verified diagnosis of AF among those who had received the QCVD were included for validation of the AF question. The same participants were included in subgroup analyses of sex, age, and type of AF. Statistical significance testing of contingency tables was performed by Fisher's exact test or $\chi^{2}$ with Yates's correction as appropriate. Confidence intervals were calculated using exact confidence intervals, computed by the method of Clopper and Pearson. ${ }^{11}$ Two-sided $P$-values with $P<0.05$ were considered as statistically significant. Analyses were performed using IBM SPSS Statistics 19 and GraphPad Prism 6.

\section{Results}

Characteristics of the study population, and the subgroup that received QCVD are shown in Table 1. Except a lower proportion of participants on antihypertensive medication,

Table I Baseline characteristics of the three study municipalities compared to the general HUNT3 population and those who received QCVD

\begin{tabular}{llll}
\hline & Study & All HUNT3 & Received \\
Baseline characteristics & participants & participants & QCVD \\
\hline Age at participation, years & $52.6 \pm 16.1$ & $52.6 \pm 16.1$ & $64.4 \pm 12.9$ \\
Age $\geq 70$ years, n (\%) & $2,565(15.8)$ & $8,158(16.1)$ & $1,515(36.6)$ \\
Height, cm & $170.6 \pm 9.2$ & $170.5 \pm 9.3$ & $169.0 \pm 9.3$ \\
Weight, kg & $79.0 \pm 15.1$ & $79.2 \pm 15.2$ & $81.7 \pm 15.3$ \\
Male sex, n (\%) & $7,406(45.6)$ & $23,049(45.4)$ & $2,052(49.5)$ \\
Myocardial infarction, n (\%) & $517(3.2)$ & $1,637(3.2)$ & $515(12.4)$ \\
Antihypertensives, n (\%) & $3,111(19.2)$ & $10,608(20.9)$ & $3,104(74.9)$ \\
Angina pectoris, n (\%) & $576(3.6)$ & $1,818(3.6)$ & $574(13.9)$ \\
Heart failure, n (\%) & $194(1.2)$ & $612(1.2)$ & $193(4.7)$ \\
Stroke, n (\%) & $431(2.7)$ & $1,298(2.6)$ & $426(10.3)$ \\
Renal disease, n (\%) & $380(2.3)$ & $1,293(2.5)$ & $379(9.2)$ \\
Diabetes mellitus, n (\%) & $688(4.2)$ & $2,189(4.3)$ & $451(10.9)$ \\
Current daily smoking, n (\%) & $2,819(17.4)$ & $8,585(16.9)$ & $597(14.4)$ \\
\hline Note:
\end{tabular}

Note: Values are mean \pm SD or $n(\%)$.

Abbreviations: QCVD, cardiovascular-specific questionnaire; HUNT3, third survey of the HUNT Study; SD, standard deviation. 
the study population was similar to the general HUNT3 population. Review of hospital and PC records revealed that a total of 502 persons among the 16,247 participants had a verified diagnosis of AF before they attended the field stations (Figure 1).

\section{The HUNT3 questionnaires' ability to identify AF}

In the study sample of 16,247 participants, 249 out of 376 persons with self-reported AF (PPV of 66.2\%) had information in their medical records that confirmed the diagnosis (Table 2), but 56 of those did not have a coded AF diagnosis in hospital discharge registers. Among the 253 persons who did not confirm their verified diagnosis of AF in the questionnaires, 65 had not received the QCVD due to wrong answers on inclusion questions, 59 had received the questionnaire but did not return it, 42 had returned it without answering the AF question, and 87 had answered "no" to the AF question (Figure 1). Of the 249 participants that confirmed their verified diagnosis of AF in QCVD, 102 answered "no" to having "other heart diseases" in the general questionnaire, indicating that a large proportion of the participants did not consider AF as a heart disease. The sensitivity of finding study participants with a verified AF diagnosis with this approach was $49.6 \%$ (Tables 2 and 3 ).

In subgroup analyses, the PPV was significantly higher and the NPV lower in males than females. The sensitivity,

Table 2 Self-reported vs verified AF diagnoses in all HUNT3 participants and the subgroup that received QCVD

\begin{tabular}{lllll}
\hline & \multicolumn{4}{c}{ Verified AF diagnosis } \\
\cline { 2 - 5 } Self-reported AFa in all & & Yes & No & Total \\
\cline { 2 - 5 } participants & Yes & 249 & 127 & 376 \\
& No & 253 & 15,618 & 15,871 \\
\multirow{2}{*}{ Self-reported AF in those } & Total & 502 & 15,745 & 16,247 \\
who received QCVD & 249 & 127 & 376 \\
& No & 188 & 3,578 & 3,766 \\
& Total & 437 & 3,705 & 4,142 \\
\hline
\end{tabular}

Notes: aself reporting of AF was a two step process. The QCVD including the AF question was given only to participants reporting cardiovascular or renal disease in the general questionnaire.

Abbreviations: AF, atrial fibrillation; QCVD, cardiovascular-specific questionnaire; HUNT3, third survey of the HUNT Study. specificity, and NPV in this study population were significantly lower in persons above 70 years of age than among younger persons, while the PPV was higher in those aged 70 years or older (Table 4). The sensitivity of self-reported $\mathrm{AF}$ was higher in permanent compared to nonpermanent $\mathrm{AF}$ (59.4\% [95\% confidence interval $\{\mathrm{CI}\} 52.5-66.1]$ vs $45.0 \%$ [95\% CI 39.0-51.2], $P=0.002$ ).

\section{The ability of the question "Has a doctor told you that you have atrial fibrillation?" to identify $A F$ in a high-risk population}

When analyzing only the 4,142 persons who actually received the QCVD, the sensitivity of finding study participants with a verified AF diagnosis with the question "Has a doctor told you that you have atrial fibrillation?" was $57.0 \%$ (Tables 2 and 3). Among the 2,996 persons that actually answered the AF question, the sensitivity was $74.1 \%$ (95\% CI 69.1-78.7), specificity 95.2\% (95\% CI 94.4-96.0), PPV 66.2\% (95\% CI 61.2-71.0), and NPV 96.7\% (95\% CI 95.9-97.3). Of the 127 persons who answered yes to the $\mathrm{AF}$ question, without having a verified AF diagnosis, 32 had other supraventricular tachycardia, 21 had palpitations, ten had frequent supraventricular or ventricular premature beats, and five had ventricular tachycardia. In 59 persons, no information of arrhythmias was found in medical records either in hospitals or PC centers (Table 5). The medical records at St Olav's Hospital of patients with self-reported AF and no AF diagnosis in either local hospitals or PC centers were also reviewed, and no more AF diagnoses were found.

In subgroup analyses on verified $\mathrm{AF}$ in this study population, the PPV of self-reported AF was significantly higher and the NPV lower in males than in females in those who received QCVD. The sensitivity and NPV were significantly lower in persons above 70 years of age than those below, while the PPV was higher in those aged 70 years or more (Table 4).

\section{The ability of hospital discharge diagnoses to identify AF}

Of the HUNT3 participants from the three study municipalities, 418 had received a diagnosis of AF in hospital discharge

Table 3 HUNT3 questionnaires' ability to identify verified AF diagnoses, compared to hospital-diagnosed AF

\begin{tabular}{lllll}
\hline Methods of AF identification & Sensitivity (95\% Cl) & Specificity (95\% CI) & PPV (95\% CI) & NPV (95\% Cl) \\
\hline HUNT3 questionnaires - all participants & $49.6(45.1-54.1)$ & $99.2(99.0-99.3)$ & $66.2(61.2-71.0)$ & $98.4(98.2-98.6)$ \\
"AF question" - those who received QCVD & $57.0(52.2-61.7)$ & $96.6(95.9-97.1)$ & $66.2(61.2-71.0)$ & $95.0(94.3-95.7)$ \\
Hospital discharge diagnoses of AF & $73.7(69.6-77.5)$ & $99.7(99.6-99.8)$ & $88.5(85.1-91.4)$ & $99.2(99.0-99.3)$ \\
\hline
\end{tabular}

Abbreviations: AF, atrial fibrillation; $\mathrm{Cl}$, confidence interval; NPV, negative predictive value; PPV, positive predictive value; QCVD, cardiovascular-specific questionnaire; HUNT3, third survey of the HUNT Study. 
Table 4 Validation of self-reported AF and hospital discharge diagnoses of $A F$ in subgroups according to sex and age with comparisons within subgroups and between self-reported and hospital-diagnosed AF

\begin{tabular}{|c|c|c|c|c|}
\hline $\begin{array}{l}\text { Validation of AF } \\
\text { diagnosis - subgroups }\end{array}$ & Sensitivity & Specificity & PPV & NPV \\
\hline \multicolumn{5}{|l|}{ Female sex } \\
\hline $\begin{array}{l}\text { HUNT3 questionnaires } \\
\text { - all participants }\end{array}$ & 44. $1^{f}$ & $99.2^{f}$ & $54 . I^{c, f}$ & $98.8^{\mathrm{c}, \mathrm{f}}$ \\
\hline $\begin{array}{l}\text { "AF question" - those } \\
\text { who received QCVD }\end{array}$ & $52.0^{f}$ & $96.5^{f}$ & $54 . I^{c, f}$ & $96.2^{c, f}$ \\
\hline $\begin{array}{l}\text { Hospital discharge } \\
\text { diagnoses of AF }\end{array}$ & 74.6 & $99.8^{\mathrm{a}}$ & 88.0 & $99.5^{c}$ \\
\hline \multicolumn{5}{|l|}{ Male sex } \\
\hline $\begin{array}{l}\text { HUNT3 questionnaires } \\
\text { - all participants }\end{array}$ & $52.5^{f}$ & $99.2^{\mathrm{e}}$ & $74.3^{f}$ & $97.8^{f}$ \\
\hline $\begin{array}{l}\text { "AF question" - those } \\
\text { who received QCVD }\end{array}$ & $59.6^{f}$ & $96.7^{f}$ & $74.3^{f}$ & $93.6^{f}$ \\
\hline $\begin{array}{l}\text { Hospital discharge } \\
\text { diagnoses of AF } \\
\text { Age } \geq \mathbf{7 0} \text { years }\end{array}$ & 73.2 & 99.6 & 88.8 & 98.8 \\
\hline $\begin{array}{l}\text { HUNT3 questionnaires } \\
\text { - all participants }\end{array}$ & $44.9^{\mathrm{b}, \mathrm{f}}$ & $98.1^{c, d}$ & $77.4^{\mathrm{c}, \mathrm{e}}$ & $92.6^{\mathrm{c}, \mathrm{f}}$ \\
\hline $\begin{array}{l}\text { "AF question" - those } \\
\text { who received QCVD }\end{array}$ & $52.2^{\mathrm{b}, \mathrm{f}}$ & $96.6^{f}$ & $77.4^{\mathrm{c}, \mathrm{f}}$ & $90 . I^{c, f}$ \\
\hline $\begin{array}{l}\text { Hospital discharge } \\
\text { diagnoses of } \mathrm{AF}\end{array}$ & 73.1 & $99.0^{c}$ & $91.4^{\mathrm{a}}$ & $96.3^{c}$ \\
\hline Age $<70$ years & & & & \\
\hline $\begin{array}{l}\text { HUNT3 questionnaires } \\
\text { - all participants }\end{array}$ & $57.6^{f}$ & $99.4^{f}$ & $55.8^{f}$ & $99.4^{e}$ \\
\hline $\begin{array}{l}\text { "AF question" - those } \\
\text { who received QCVD }\end{array}$ & 65.0 & $96.6^{f}$ & $55.8^{f}$ & $97.7^{f}$ \\
\hline $\begin{array}{l}\text { Hospital discharge } \\
\text { diagnoses of } \mathrm{AF}\end{array}$ & 74.7 & 99.8 & 84.0 & 99.7 \\
\hline
\end{tabular}

Notes: ${ }^{a} P<0.05,{ }^{b} P<0.01,{ }^{c} P<0.00$ I for male vs female sex and age over vs under 70 years. ${ }^{d} P<0.05,{ }^{e} P<0.0$ I, ${ }^{f} P<0.00$ I for hospital-diagnosed vs self-reported $A F$ in each subgroup.

Abbreviations: AF, atrial fibrillation; NPV, negative predictive value; PPV, positive predictive value; QCVD, cardiovascular-specific questionnaire; HUNT3, third survey of the HUNT Study.

Table 5 Other arrhythmias and relevant diagnoses in persons with self-reported AF, but no verified AF diagnosis

\begin{tabular}{ll}
\hline Other arrhythmias/symptoms & $\mathbf{n}$ \\
\hline Sinus tachycardia & 2 \\
Atrial tachycardia & 4 \\
AVRT/WPW syndrome & 6 \\
AV-nodal reentry tachycardia & $1 \mathrm{I}$ \\
Frequent premature supraventricular complexes & 8 \\
Frequent premature ventricular complexes & 2 \\
Ventricular tachycardia & 5 \\
Palpitations & 21 \\
Tachycardia, type not specified & 9 \\
No arrhythmias & 59 \\
\hline
\end{tabular}

Abbreviations: AF, atrial fibrillation; AVRT, atrioventricular reentry tachycardia; WPW, Wolff -Parkinson -White. registers before participating in HUNT3 (Table 6). Of those, 370 had a diagnosis of AF or atrial flutter that could be confirmed by thorough evaluation of their hospital and PC medical records, which is $74 \%$ of the 502 verified AF diagnoses. Of the 370 persons with a verified diagnosis of AF, 177 had not reported AF in HUNT3 questionnaires. When including persons with a hospital diagnosis of AF within 3 and 6 months after the HUNT3 inclusion, three and eight more persons were found to have a verified AF diagnosis, respectively.

The NPV and specificity of verified hospital discharge diagnoses of AF in this study population were significantly higher in females than in males, and in those below 70 years of age than in those above 70 years (Table 4).

\section{Comparison of the validity of questionnaire-based identification and hospital discharge diagnoses of $A F$}

When comparing the use of hospital discharge diagnoses of AF to identification of AF by the HUNT3 questionnaires, hospital discharge diagnoses perform significantly better on all parameters in all subgroups, except for the sensitivity of finding patients with verified $\mathrm{AF}$ under 70 years of age (Table 4).

\section{AF that was not detected by questionnaires or retrieval of diagnoses from registers}

After a thorough review of written documentation and ECGs in medical records in hospitals and PC centers for 500 random participants, we found three persons with a diagnosis of AF that was neither self-reported as AF nor registered with an $\mathrm{AF}$ diagnosis in hospital or PC registers.

\section{Discussion}

In this validation study, retrieval of hospital discharge diagnoses of AF was superior to identification of AF by question-

Table 6 Validation of hospital discharge diagnoses of AF vs verified $A F$ diagnoses

\begin{tabular}{lllll}
\hline & \multicolumn{4}{c}{ Verified AF diagnosis } \\
\cline { 2 - 5 } & & Yes & No & Total \\
\hline Hospital discharge & Yes & 370 & 48 & 418 \\
diagnosed AF & No & 132 & 15,697 & 15,829 \\
& Total & 502 & 15,745 & 16,247 \\
\hline
\end{tabular}

Abbreviation: $\mathrm{AF}$, atrial fibrillation. 
naires. Self-reported AF substantially underestimates the true sensitivity compared with hospital and PC data.

\section{The HUNT3 questionnaires' ability to identify $A F$}

In larger epidemiological studies, there is a need to limit the number of questions to all participants by creating questionnaires targeting subgroups. In HUNT3, this was solved by handing out a general questionnaire to all participants, and further questionnaires only to those with different selfreported diseases in Q1. There was no specific question on $\mathrm{AF}$ in $\mathrm{Q} 1$, but questions on cardiovascular or renal disease as selection to the QCVD. We found poor results of this approach for identification of AF, with a study population sensitivity of $50 \%$, and a PPV of $66 \%$. Including a specific question on AF in the general questionnaire could have improved sensitivity. Our results also identify the challenge with lower answering rates and more incompletely answered forms for follow-up questionnaires. Of the participants that did not confirm their AF diagnosis in the HUNT3 questionnaires, $40 \%$ had received QCVD, but either did not return the questionnaire, or returned it without answering the AF question.

\section{Validation of the question "Has a doctor told you that you have AF?"}

When restricting analyses to those having answered the question on AF, the study population sensitivity was only $57 \%$, and the PPV was also rather low. Many participants seemed to have mistaken other arrhythmias and symptoms with AF. One should also be aware that these results are obtained in a questionnaire that was handed out to participants with selfreported cardiovascular or renal disease, with a subsequent higher prevalence of AF. A total of 87 participants who answered "no" to the AF question had actually received a diagnosis of AF in hospital or PC before attending HUNT3. This underlines the uncertainty in using self-reported AF as an endpoint.

\section{Discharge diagnoses of AF in hospitals}

Despite a rather low sensitivity in this study population, AF diagnoses retrieved from hospital discharge registries will be sufficient for many health-survey studies. There is often a delay from registration of AF diagnosis in PC centers to the hospital registration. An increased sensitivity will be achieved by increasing the time for retrieval by 3 or more months after the participation date. However, the medical records should then be reviewed to determine whether the first episode of AF was before or after the study inclusion date.

\section{Identification of AF cases by questionnaires compared to retrieval of hospital discharge diagnoses}

In this study, a diagnosis of AF in hospital discharge registries has a study population sensitivity of $74 \%$ and a PPV of $89 \%$, which is superior to self-reported AF from questionnaires. Reviewing written documentation and ECGs from medical records will improve the specificity. However, data from hospital-diagnosed AF will underestimate the prevalence in the population substantially and supplementary methods should be considered. Nondiagnosed AF and underreporting of $\mathrm{AF}$ in registries are major limitations to a true estimate of AF prevalence. A study using an implantable cardiac monitor in patients with cryptogenic stroke found that $30 \%$ had AF after 36 months, compared to $3 \%$ of those without an implantable cardiac monitor. ${ }^{12}$ In a 2 -week screening study of 75-76-year-old persons in Sweden, 3\% of those were found to have previously unknown AF, in addition to the $9.3 \%$ having known AF. ${ }^{13}$ This implies that $20 \%-25 \%$ of AF cases in the elderly are undiagnosed. The magnitude of undiagnosed AF in younger persons has not been studied in detail, but three out of 1,296 persons without known cardiac disease, hypertension, or diabetes who underwent echocardiography in HUNT3 had AF during examination that had not been previously diagnosed. ${ }^{14}$ In addition to undiagnosed AF, there is also underreporting of known AF in registries. Out of 500 random persons without AF in HUNT3 questionnaires or hospital and $\mathrm{PC}$ diagnosis registries, three persons were found to have a verified diagnosis of AF after full review of their medical records. The estimate of unrecognized AF in such small samples is uncertain, and the magnitude of undiagnosed AF in middle-aged persons is unknown. It is therefore difficult to estimate this effect precisely, but our results clearly overestimate the true sensitivity, and from the findings discussed earlier, a $10 \%-20 \%$ overestimation of the true sensitivity is possible. However, this should not affect the comparisons of the two methods for AF diagnoses in the present study, as the persons with a false-negative AF status will affect the results of both groups similarly.

\section{Subgroup analyses}

There are significant differences regarding study population PPV and NPV in subgroups that can be attributed to a lower prevalence of AF in females and persons less than 70 years of age. A study population sensitivity of self-reported AF below $50 \%$ in participants over 70 years of age is an important finding. As the prevalence of AF increases steeply with older age, using self-reported AF will result in a high number of 
missed AF cases in older populations. The study population specificity of self-reported AF is also significantly lower in this group than in participants under 70 years of age.

\section{Comparisons to other studies}

Although self-reported AF is often used as an endpoint, ${ }^{15-20}$ few studies have made attempts to validate this approach. The AF diagnosis was validated in a subgroup of 400 persons with self-reported AF in the Physicians' Health Study. Documentation in medical records was missing in $\sim 44 \%$ of the participants, and the diagnosis of AF was not supported in $7 \%$, making the results uncertain. Furthermore, the sensitivity of the question was not reported. The same question will probably give considerably poorer results in a general population. Psaty et al analyzed the hospital discharge summary of $10 \%$ of their participants with self-reported AF, and found no registration of AF in any of those reports. ${ }^{21}$ Conen et $\mathrm{al}^{20}$ reported that $10 \%$ of AF patients are asymptomatic, and Svennberg et $\mathrm{al}^{13}$ reported that $3.0 \%$ of a screened $75 / 76$-yearold population had undiagnosed AF, limiting the sensitivity of self-reported AF.

Multiple studies have estimated the prevalence of AF from hospital discharge diagnoses of $\mathrm{AF}^{4,22,23}$ Jensen et al published a review of validated methods for identifying AF using administrative data. ${ }^{8}$ They reported a median sensitivity of $79 \%$ and a median PPV of $89 \%$ in 16 different studies. Most of those studies used only inpatient data, and many are 10-15 years old, with a slightly different prevalence and other treatment strategies for AF than today. The study population sensitivity in our study is somewhat lower than reported by Jensen et al, while the PPV is the same. We have performed a more systematic search for AF cases, both in hospitals and in PC centers, in addition to using self-reported AF and possibly identified more individuals with $\mathrm{AF}$, resulting in a lower study population sensitivity for hospital-diagnosed AF.

Of more recent studies, Norberg et al had a similar approach to the present study by including hospitaldiagnosed, and PC-diagnosed $\mathrm{AF}^{22}$ In addition, they analyzed ECGs from a regional database. They reported a higher precision for ICD-10-diagnosed AF than earlier studies, with a sensitivity of $93 \%$ and a PPV of $96 \%$, explained by using AF diagnoses from both hospitals and PC centers to find AF cases. In addition, their only false-negative cases were persons with an ECG showing AF, but no AF diagnosis in the registries. The numbers for hospital discharge diagnoses of $\mathrm{AF}$ alone were not reported, which are the most relevant for other studies, as few regions have easy access to all AF diagnoses from $\mathrm{PC}$ centers. Even if we included AF diagnoses from both hospitals and PC centers, the sensitivity is lower in our study as we also included persons with self-reported AF. These are persons that might have received the diagnosis in other regions of the country or the diagnosis has been forgotten to be coded by physicians. The higher PPV in the study by Norberg et al reflects a higher accuracy of coding practice, which may be explained by increased awareness of AF by both physicians and patients in more recent studies. This discrepancy underlines the importance of regional and temporal differences in treatment strategies, registration, and handling of patients when assessing data from AF registries. To our knowledge, no previous studies have validated selfreported AF and hospital discharge diagnoses of AF in the same population.

\section{Strengths and limitations}

PC diagnoses of AF have to our knowledge not been validated previously, and this was also not the aim of this study. Including other PC diagnoses like palpitations and other supraventricular arrhythmias might have improved the precision of our estimates, but would have increased the number of records needed to review to a large extent, and were therefore not included. Restricting the search in PC to ICPC code K78 possibly results in a slightly higher number of false-negative AF cases, and contributes to overestimating especially the sensitivity of self-reported and hospital-diagnosed AF.

Compared to the population invited to HUNT3, the participants have been shown to have a slightly lower prevalence of chronic diseases, including cardiovascular diseases, higher socioeconomic status, and a lower mortality than nonparticipants. ${ }^{24}$ As the difference is minor, this should not affect the results of hospital-diagnosed AF to a large extent.

In HUNT3, the AF question is answered only by persons with self-reported hypertension or cardiovascular or renal disease. This group has a higher prevalence of AF, influencing the results. The lower response rate due to two questionnaires also has a negative impact. Our results for self-reported AF, therefore, cannot be generalized to the normal population with an expected lower prevalence of AF and a higher proportion of persons with other arrhythmias.

There are persons with AF, especially among the elderly with multiple comorbidities, who are not captured by registers in hospitals or PC centers. The elderly also have a lower sensitivity for self-reported AF. The values for the study population sensitivity reported are therefore higher than the expected real-life values. An increased knowledge of AF in the population and validation of self-reported AF are needed to increase the value of self-reported disease. When a scanned 
ECG was lacking, we had to rely upon written descriptions of ECG, which may have caused misclassifications. But after reviewing medical records, only 17 participants were left with an indefinite diagnosis.

The strengths of this study are the detailed review of medical records and diagnoses in both hospitals and PC centers, with an available ECG or sufficient written analysis of ECG in most cases. We also included self-reported AF, and did a thorough review of medical records in a subset of persons without information on AF in registries or HUNT3, to characterize the ability of the different methods in capturing persons with AF. This is, to our knowledge, the first study to compare self-reported and hospital-diagnosed AF.

\section{Conclusion}

Use of questionnaires alone to define cases of $\mathrm{AF}$ has low sensitivity and specificity compared with hospital and PC data. Hospital discharge diagnoses of AF are acceptable for many epidemiological studies and should be preferred. When a high level of sensitivity is required, PC registries should also be included, but there is still a subset of AF patients that are missed and additional approaches should be considered to find those.

\section{Acknowledgments}

This study was supported by grants from the Norwegian Council on Cardiovascular Diseases, the Liaison Committee between the Central Norway Regional Health Authority and the Norwegian University of Science and Technology, and the Department of Neuroscience at the Norwegian University of Science and Technology. The authors also want to thank the R\&D department of the Nord-Trøndelag Health Trust and the general practitioners supporting the local data collection.

\section{Disclosure}

The authors report no conflicts of interest in this work.

\section{References}

1. Wolowacz SE, Samuel M, Brennan VK, Jasso-Mosqueda JG, Van Gelder IC. The cost of illness of atrial fibrillation: a systematic review of the recent literature. Europace. 2011;13(10):1375-1385.

2. Ball J, Carrington MJ, McMurray JJ, Stewart S. Atrial fibrillation: profile and burden of an evolving epidemic in the 21 st century. Int $J$ Cardiol. 2013;167(5):1807-1824.

3. Camm AJ, Kirchhof P, Lip GY, et al. Guidelines for the management of atrial fibrillation: the Task Force for the Management of Atrial Fibrillation of the European Society of Cardiology (ESC). Eur Heart J. 2010;31(19):2369-2429.
4. Friberg L, Bergfeldt L. Atrial fibrillation prevalence revisited. J Int Med. 2013;274(5):461-468.

5. Wilke T, Groth A, Mueller S, et al. Incidence and prevalence of atrial fibrillation: an analysis based on 8.3 million patients. Europace. 2013;15(4):486-493.

6. Chen LY, Shen W-K. Epidemiology of atrial fibrillation: a current perspective. Heart Rhythm. 2007;4(3, Suppl 1):S1-S6.

7. Chugh SS, Havmoeller R, Narayanan K, et al. Worldwide epidemiology of atrial fibrillation: a Global Burden of Disease 2010 Study. Circulation. 2014;129(8):837-847.

8. Jensen PN, Johnson K, Floyd J, Heckbert SR, Carnahan R, Dublin S. A systematic review of validated methods for identifying atrial fibrillation using administrative data. Pharmacoepidemiol Drug Saf. 2012;21(Suppl 1):141-147.

9. Krokstad S, Langhammer A, Hveem K, et al. Cohort profile: the HUNT Study, Norway. Int J Epidemiol. 2013;42(4):968-977.

10. Calkins H, Kuck KH, Cappato R, et al. 2012 HRS/EHRA/ECAS Expert Consensus Statement on Catheter and Surgical Ablation of Atrial Fibrillation: recommendations for patient selection, procedural techniques, patient management and follow-up, definitions, endpoints, and research trial design. Europace. 2012;14(4):528-606.

11. Clopper C, Pearson ES. The use of confidence or fiducial limits illustrated in the case of the binomial. Biometrika. 1934;26(4):404-413.

12. Thijs VN, Brachmann J, Morillo CA, et al. Predictors for atrial fibrillation detection after cryptogenic stroke: results from CRYSTAL AF. Neurology. 2016;86(3):261-269.

13. Svennberg E, Engdahl J, Al-Khalili F, Friberg L, Frykman V, Rosenqvist M. Mass screening for untreated atrial fibrillation: The STROKESTOP Study. Circulation. 2015;131(25):2176-2184.

14. Dalen H, Thorstensen A, Aase SA, et al. Segmental and global longitudinal strain and strain rate based on echocardiography of 1266 healthy individuals: the HUNT study in Norway. Eur J Echocardiogr. 2010;11(2):176-183.

15. Myrstad M, Lochen ML, Graff-Iversen S, et al. Increased risk of atrial fibrillation among elderly Norwegian men with a history of long-term endurance sport practice. Scand J Med Sci Sports. 2014;24(4):e238-e244.

16. Aizer A, Gaziano JM, Cook NR, Manson JE, Buring JE, Albert CM. Relation of vigorous exercise to risk of atrial fibrillation. Am J Cardiol. 2009;103(11):1572-1577.

17. Goren A, Liu X, Gupta S, Simon TA, Phatak H. Quality of life, activity impairment, and healthcare resource utilization associated with atrial fibrillation in the US National Health and Wellness Survey. PLoS One. 2013;8(8):e71264.

18. Williams PT, Franklin BA. Reduced incidence of cardiac arrhythmias in walkers and runners. PLoS One. 2013;8(6):e65302.

19. Soliman EZ, Howard G, Meschia JF, et al. Self-reported atrial fibrillation and risk of stroke in the Reasons for Geographic and Racial Differences in Stroke (REGARDS) study. Stroke. 2011;42(10):2950-2953.

20. Conen D, Tedrow UB, Cook NR, Moorthy MV, Buring JE, Albert CM. Alcohol consumption and risk of incident atrial fibrillation in women. JAMA. 2008;300(21):2489-2496.

21. Psaty BM, Manolio TA, Kuller LH, et al. Incidence of and risk factors for atrial fibrillation in older adults. Circulation. 1997;96(7):2455-2461.

22. Norberg J, Backstrom S, Jansson JH, Johansson L. Estimating the prevalence of atrial fibrillation in a general population using validated electronic health data. Clin Epidemiol. 2013;5:475-481.

23. Smith JG, Platonov PG, Hedblad B, Engstrom G, Melander O. Atrial fibrillation in the Malmo Diet and Cancer study: a study of occurrence, risk factors and diagnostic validity. Eur J Epidemiol. 2010;25(2):95-102.

24. Langhammer A, Krokstad S, Romundstad P, Heggland J, Holmen J. The HUNT study: participation is associated with survival and depends on socioeconomic status, diseases and symptoms. BMCMed Res Methodol. 2012;12:143. 
Clinical Epidemiology

\section{Publish your work in this journal}

Clinical Epidemiology is an international, peer-reviewed, open access, online journal focusing on disease and drug epidemiology, identification of risk factors and screening procedures to develop optimal preventative initiatives and programs. Specific topics include: diagnosis, prognosis, treatment, screening, prevention, risk factor modification,

Submit your manuscript here: https://www.dovepress.com/clinical-epidemiology-journal
Dovepress

systematic reviews, risk and safety of medical interventions, epidemiology and biostatistical methods, and evaluation of guidelines, translational medicine, health policies and economic evaluations. The manuscript management system is completely online and includes a very quick and fair peer-review system, which is all easy to use. 